\title{
Clinical, Morphological, and Molecular Study of Diffuse WHO Grade II and III Astrocytomas: A Retrospective Analysis from a Single Tertiary Care Institute
}

\author{
Ramya Lakshmi Veduruvada ${ }^{1}$ Megha S. Uppin ${ }^{1}$ Meher Lakshmi Konatam² Rajesh Alugolu ${ }^{30}$ \\ Vamsi Krishna Yeramneni ${ }^{3}$ Suchanda Bhattacharjee ${ }^{30}$ Mudumba Vijaya Saradhi ${ }^{3}$ \\ Monica Malik Irukulla ${ }^{4}$ Madhumohan Rao ${ }^{5}$ Nagaraj Velugonda6(0)
}

${ }^{1}$ Department of Pathology, Nizam's Institute of Medical Sciences, Punjagutta, Hyderabad, Telangana, India

2 Department of Medical Oncology, Nizam's Institute of Medical Sciences, Punjagutta, Hyderabad, Telangana, India

${ }^{3}$ Department of Neurosurgery, Nizam's Institute of Medical Sciences,

Punjagutta, Hyderabad, Telangana, India

${ }^{4}$ Department of Radiation Oncology, Nizam's Institute of Medical

Sciences, Punjagutta, Hyderabad, Telangana, India

${ }^{5}$ Stem Cell Facility and Regenerative Medicine, Nizam's Institute of

Medical Sciences, Hyderabad, Telangana, India

${ }^{6}$ Department of Medical Oncology, Nizam's Institute of Medical

Sciences, Hyderabad, Telangana, India

Ind J Med Paediatr Oncol 2021;42:569-576.

\author{
Address for correspondence Megha S. Uppin, MD, Additional \\ Professor, Department of Pathology, Nizam's Institute of Medical \\ Sciences, Punjagutta, Hyderabad, Telangana-500082, India \\ (e-mail: megha_harke@yahoo.co.in).
}

\begin{abstract}
Keywords

- glioma

- diffuse astrocytoma

- immunohistochemistry

- IDH1/2 mutations

- Sanger sequencing

- survival

Introduction Astrocytomas are the most common gliomas, classified on the basis of grade and IDH mutation status according to the World Health Organization (WHO) 2016 update. IDH mutations are seen in 70 to $80 \%$ of diffuse grade II and III astrocytomas and are associated with better outcome. They serve as predictive biomarker in IDH-targeted therapies such as small-molecule inhibitors or vaccines.

Objective The aim of this study was to analyze the clinical, morphological, immunohistochemical, and molecular genetic characteristics of diffuse astrocytoma (DA: grades II and III). The IDH mutant and wild-type tumors are compared and contrasted with survival analysis on follow-up.

Materials and Methods This was a retrospective study conducted on surgically resected tumor specimens. The hematoxylin and eosin-stained slides were examined for histologic features. Immunohistochemistry $(\mathrm{IHC})$ was performed using IDH1R132H, ATRX, p53, and Ki67. All cases of negative immunohistochemical expression of IDH1R132H were subjected to IDH1 mutation analysis by Sanger sequencing. Overall survival was estimated by the Kaplan-Meier method using the log-rank (Mantel-Cox) test.
\end{abstract}

DOI https://doi.org/ 10.1055/s-0041-1741061. ISSN 0971-5851. (c) 2021. Indian Society of Medical and Paediatric Oncology. All rights reserved.

This is an open access article published by Thieme under the terms of the Creative Commons Attribution-NonDerivative-NonCommercial-License, permitting copying and reproduction so long as the original work is given appropriate credit. Contents may not be used for commercial purposes, or adapted, remixed, transformed or built upon. (https://creativecommons.org/ licenses/by-nc-nd/4.0/)

Thieme Medical and Scientific Publishers Pvt. Ltd., A-12, 2nd Floor, Sector 2, Noida-201301 UP, India 
Results The study included 51 cases of DA in the age of 17 to 66 years, mean \pm standard deviation was $35.5 \pm 9.7$ years, and male:female ratio was 2:1.The IDH1R132H cytoplasmic immunopositivity was seen in 36 cases (70.5\%), of which $63.6 \%$ were of grade II and $72.5 \%$ were of grade III. ATRX showed loss of expression in 50 cases (98\%), and $p 53$ showed diffuse strong immunohistochemical expression in all the cases of IDH mutant tumors. The difference in the age at presentation for IDH mutant (32.5 years) and wild type tumors (38 years) was statistically significant. Median survival was 55.3 months and 22.2 months in of IDH mutant and wild type cases, respectively.

Conclusion IHC and sequencing for IDH mutations is helpful in making an integrated diagnosis and classifying definite molecular subgroups of astrocytic tumors. Mutations in IDH core-elate with survival. IDH mutant tumors showed longer survival duration and are good prognostic indicators.

\section{Introduction}

The World Health Organization (WHO) 2016 update brought in paradigm changes in understanding and classification of central nervous system neoplasms bringing in the molecular biology of these tumors. ${ }^{1}$ The adult diffuse gliomas are essentially classified on the basis of IDH mutations, which have been observed to be an important prognostic and predictive biomarker. ${ }^{1-3}$ IDH mutations are reported in $>80 \%$ of WHO grade II/III gliomas. ${ }^{4}$ Other studies have reported IDH mutations in $72 \%$ of grade II and $64 \%$ of grade III astrocytomas. ${ }^{5,6}$ Diffuse gliomas that do not harbor IDH mutations exhibit aggressive behavior irrespective of the grade of the tumor. IDH wild-type diffuse astrocytoma (DA) and anaplastic astrocytoma (AA) have a survival similar to or only slightly longer than $I D H$ wildtype glioblastoma. ${ }^{2-4,7,8}$ Presence of EGFR amplification and a genotype of $7 q$ gain and $10 q$ loss have been associated with worse outcome. ${ }^{9}$ Study of $I D H$ mutations alone in diffuse glioma is an important research tool for targeted therapies and possible vaccines.

In this article, we share our experience with diffuse astrocytic tumors, WHO grades II and III, with respect to clinical features, histopathology, and molecular studies. The survival was compared between two molecular subgroups.

\section{Materials and Methods}

Study Design: This was a retrospective evaluation of the clinical, histopathological, and molecular markers of WHO grade II and III astrocytomas. The IDH mutant and wild-type tumors have been compared with respect to survival.

Study Setting: The study was performed in a tertiary care center. The study period was from January 2015 to June 2017.

Participants: All patients diagnosed on histopathology as grade II or III astrocytoma were included in all age groups. Other types of gliomas were excluded.

Variables: The astrocytomas were classified into IDH mutant and wild-type gliomas. The two molecular types were compared with respect to demographic features, location, survival, and outcome.
Primary Outcome: Comparison of the longest survival period between IDH mutant and wild-type tumors.

Secondary Outcome: Comparison of other variables like age, location, and histopathology between IDH mutant and wildtype tumors.

Data Collection: The patient details including demographic features, clinical presentation, and radio imaging findings were obtained from the request forms. The treatment details were obtained from medical records. The duration of the follow-up was calculated on the basis of last followup date or death of the patient, whichever was earlier.

\section{Histopathology}

The tumor specimens were fixed in formalin and processed routinely. The hematoxylin and eosin-stained slides were examined for histologic features of DA and categorized into grade II or III as per the WHO 2016 Classification. ${ }^{1}$

\section{Immunohistochemistry (IHC)}

This was performed using IDH1R132H (clone D09, Dianova, dilution 1:200), ATRX (HPA001906, Sigma Aldrich, dilution 1:500), p53 (Clone D07, Bio-Genex), and Ki67 (Sigma Aldrich, Ready to Use). Cytoplasmic staining for IDH1R132H and nuclear staining for $A T R X, p 53$, and Ki67 were interpreted as positive

\section{Sequencing}

All cases of negative IDH1R132H immunoexpression were subjected to IDH mutation analysis by Sanger sequencing.

\section{DNA Extraction FFPE Tissue}

Formalin-fixed, paraffin-embedded (FFPE) blocks were cut into 12 serial sections of $18 \mu \mathrm{m}$ each. Areas consisting of at least $80 \%$ tumor cells were marked on the unstained sections that were then scraped into polypropylene tubes and deparaffinized using xylene. The collected tumor tissue was subjected to deoxyribonucleic acid (DNA) extraction with the QIAmp DNA FFPE Tissue kit following manufacturer's protocol. Isolated DNA concentration was quantified by spectrophotometry using Nano drop 2000 instrument. 
Table 1 Molecular subgroups of different astrocytomas based on IDH mutation and grade

\begin{tabular}{|l|l|l|l|l|}
\hline & $\begin{array}{l}\text { IDH mutant } \\
\text { astrocytoma, } \\
\text { WHO grade II } \\
(\mathrm{N}=7)\end{array}$ & $\begin{array}{l}\text { IDH wild type astrocytoma, } \\
\text { WHO grade II (N=4) }\end{array}$ & $\begin{array}{l}\text { IDH mutant } \\
\text { astrocytoma, } \\
\text { WHO grade III } \\
(\mathrm{N}=31)\end{array}$ & $\begin{array}{l}\text { IDH wild type } \\
\text { astrocytoma, } \\
\text { WHO grade III (N=9) }\end{array}$ \\
\hline Age group (years) & $17-40$ & $29-45$ & $18-66$ & $25-55$ \\
\hline Male:female ratio & $0.75: 1$ & $3: 1$ & $3.8: 1$ & $0.9: 1$ \\
\hline Common location & $\begin{array}{l}\text { Frontal and } \\
\text { temporal lobes }\end{array}$ & Temporal & Frontal & Frontal \\
\hline Mean age & 29 years & 38 years & 36 years & 38 years \\
\hline Overall survival (months) & 63 & N/A & 60 & 90 \\
\hline Median survival (months) & 60 & N/A & 56 & 38 \\
\hline
\end{tabular}

Abbreviations: N/A, not available; WHO, World Health Organization.

\section{IDH Mutation Analysis}

Polymerase chain reaction (PCR) amplification of the target region was performed by mixing $50 \mathrm{ng}$ of extracted tumor DNA as template, $10 \mu \mathrm{L}$ of HotStar Taq 2X Mastermix (DSS Takara), $200 \mathrm{nM}$ of the respective forward and reverse primers, and high-purity water to a final volume of $20 \mu \mathrm{L}$ (IDH1 PCR primers: forward, AATGAGCTCTATATGCCATCACTG; reverse, TTCATACCTTGCTTAATGGGTGT). The PCR cycling conditions included an initial denaturation step at $95^{\circ} \mathrm{C}$ for 5 minutes, followed by 40 cycles of denaturation at $95^{\circ} \mathrm{C}$ for 30 seconds, annealing at $60^{\circ} \mathrm{C}$ for 30 seconds, and elongation at $72^{\circ} \mathrm{C}$ for 30 seconds, concluding with a final elongation step at $72^{\circ} \mathrm{C}$ for 10 minutes. The PCR products were checked by electrophoresis on $2 \%$ agarose gel. Amplified PCR products were subjected to Sanger sequencing for detection of mutations in IDH1 gene.

\section{Fluorescence In Situ Hybridization}

Fluorescence in situ hybridization (FISH) was performed on FFPE sections using dual color locus specific probes for $1 p 36$ and 19q13 paired, respectively, with the reference probes for $1 q 25$ and 19p13 (Vysis). This test was performed in the study group in IDH mutant gliomas if the ATRX immunoexpression was retained. Following pretreatment, probes were added to the sections and the slides were subjected to denaturation at $78^{\circ} \mathrm{C}$ for 5 minutes and hybridization at $37^{\circ} \mathrm{C}$ for 16 hours in thermobrite chamber. After counter staining with 4',6-diamidino-2-phenylindole, slides were examined under fluorescent microscope. The hybridization signals were scored in at least 200 nonoverlapping nuclei. The ratio of $1 p / 1 q$ and $19 q / 19 p$ was calculated by taking the number of test and control signals. Also, $1: 2$ ratio of signals in $>50 \%$ cells was taken as a criteria for codeletion. ${ }^{10}$

Sample Size: The study included 51 patients. All consecutive cases diagnosed as DA WHO grades II and III were included in the study period.

\section{Statistical Analysis}

Demographic and other relevant clinical data were presented as mean or proportioned appropriately. Statistical analysis was performed using Graph Pad Prism 8.4.3. Data were analyzed and correlation was done between IDH mutation status and grading of astrocytoma. Overall survival (OS) was defined as the interval between primary surgery and death or last follow-up. OS was estimated by the Kaplan-Meier method using the log-rank (Mantel-Cox) test. A $p$-value less than 0.05 was considered to be of statistical significance.

\section{Interpretation of Testing (-Table 1)}

DA, IDH Mutant: Positive IDH1R132H IHC or mutation analysis with loss of ATRX expression and diffuse p53 immunopositivity.

DA Wild type: Negative IDH1R132H IHC and mutation analysis with loss of ATRX expression or absence of $1 p 19 q$ codeletion if the ATRX was retained.

\section{Ethics}

The study was approved by Nizam's Institute of Medical Sciences (EC/NIMS/2316/2019, dated 01.04.2019). Waiver of consent was granted since the study was retrospective and was performed on archival tissue blocks. Confidentiality was maintained by the deidentification of data. The procedures followed were in accordance with the ethical standards of the responsible committee on human experimentation and with the Helsinki Declaration of 1964, as revised in 2013.

\section{Results}

\section{Demographic, Clinical, and Radiological Data}

Total 51 cases were diagnosed as astrocytoma (WHO grades II and III) in the study period. The patients were in the age group of 17 to 66 years with mean \pm standard deviation (SD) age being $35.5 \pm 9.7$ years, with a male:female (M:F) ratio of 2:1. Of the 51 cases, 11 were WHO grade II and 40 were WHO grade III. The mean age was $31.2 \pm 8.6$ years (median 29 years) and $36.45 \pm 9.7$ years (median 33 years) for grade II and III tumors, respectively. Only two patients were less than 18 years of age with one each of grade II and grade III tumors. - Graph 1 depicts the distribution of grades within various age groups.

Most common clinical presentation of the patients was headache $(33,70 \%)$ followed by seizures $(25,53 \%)$ and 


\section{Age and sex wise distribution among Grade II and III astrocytomas}

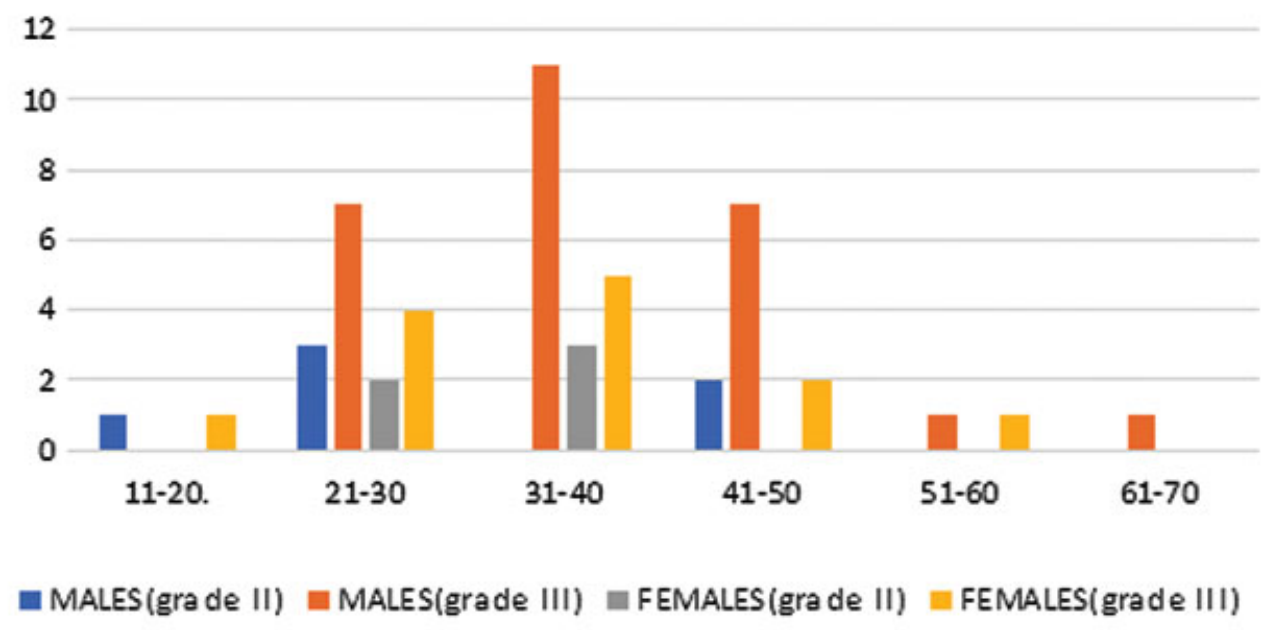

Graph 1 Age distribution among grade II and III astrocytomas.

weakness of limbs, and the most common location of tumor was frontal lobe $(14,32 \%)$.

The magnetic resonance imaging findings were reviewed and correlated with final histopathology. Thirteen cases of grade III astrocytoma were considered to be low-grade glioma due to absence of perilesional edema and contrast enhancement, whereas three cases of grade II astrocytoma were considered to be of high grade due to extensive perilesional edema and mass effect. Apart from these, there was correlation of radio imaging and histopathological features. Two cases, one each of grades II and III, had pattern of gliomatosis cerebri.

The surgical notes mentioned that $62 \%$ of the tumors had gross total resection.

\section{Histomorphology}

WHO grade II astrocytoma constituted 11/51 (21.6\%) cases. These tumors showed increased cellularity and pleomorphism without atypical mitosis. WHO grade III astrocytoma constituted 40/51 (78.4\%) cases. These tumors showed hypercellularity, pleomorphism, and atypical mitosis. Atypical mitosis was more than one per section in grade III tumors. Microcystic spaces were identified in 11/51 (21.5\%) tumors. Focal oligodendroglial pattern was observed in 2/51 (3.9\%) grade III tumors and these resembled the erstwhile oligoastrcotyomas. Tumor calcification was identified in only one (1.9\%) grade III tumor. Gemistocytes were observed in 15/51 (29.4\%) cases, of which 13 belonged to grade III. Necrosis and microvascular proliferation, which are typical features of grade IV tumors, were absent in all cases.

\section{Immunohistochemistry}

IDHR132H: The cytoplasmic immunopositivity was seen in $36 / 51$ (70.5\%) cases, of which $63.6 \%$ were grade II and $72.5 \%$ were grade III.
ATRX: The ATRX showed loss of expression in 50 cases whereas one IDH mutant AA (grade III) showed retained ATRX nuclear expression, but this case did not show $1 p 19 q$ codeletion on FISH analysis. Of the cases that were negative for IDH1R132H, five had loss of $A T R X$ expression.

p53: There was diffuse strong immunohistochemical expression of $p 53$ in all the cases of IDH mutant tumors. Among 15 tumors that were negative for IDH1R132H, 6 showed diffuse p53 expression whereas the rest 9 were negative.

Ki67: The mean \pm SD Ki67 for grade II tumors was $2.36 \pm 1.0$ (range: $1-5$ ) and grade III tumors was $15.6 \pm 11.3$ (range: 6-64).

\section{Sequencing}

Out of the 15 tumors that were negative for IDH1R132H IHC, only 2 cases (both belonging to grade III) showed mutations in Sanger sequencing as depicted in -Fig. 1. These mutations were also identified in IDH1R132H locus. None of the cases showed any other IDH1 mutations.

Applying integrated diagnosis, $74.5 \%$ tumors in this study were IDH mutant.

\section{Follow-Up Period and Survival}

Follow-up data were available in 37/51 patients. All these patients were given $54 \mathrm{~Gy}$ of intensity modulated radiotherapy (IMRT) and chemotherapy with temozolomide for a period ranging from 31 to 1,488 days and a mean of $374 \pm 269.4$ days. All the patients were followed up for a period of 2 days to 90 months. The follow-up and survival analysis were done separately for IDH mutant and IDH wildtype astrocytomas. The OS was $48.6 \%$, and $51.4 \%$ patients died at the end of follow-up period. Eighteen of the patients are alive at the longest follow-up including $14 \mathrm{IDH}$ mutant and 4 IDH wild-type patients. The OS differed in grades with $57.2 \%$ in grade II and $46.7 \%$ in grade III astrocytomas. 


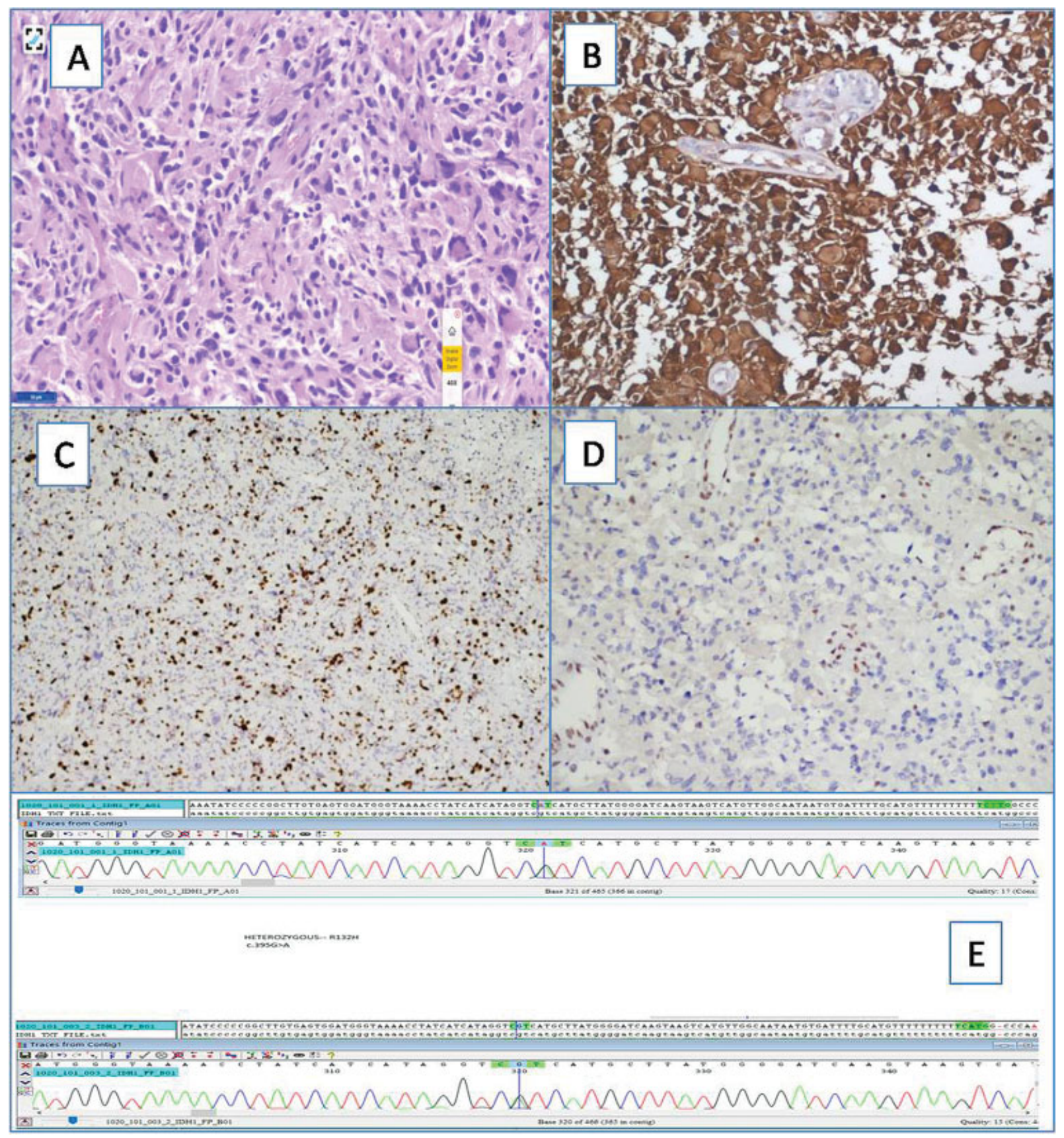

Fig. 1 (Top) Microscopic images of a grade III anaplastic astrocytoma. (A) Cellular tumor with marked pleomorphism and atypia showing many gemistocytes $(H \& E \times 40)$. (B) Cytoplasmic positivity for IDH1R132H; HRP-polymer $\times 100$. (C) Tumor cells showing diffuse strong expression of $p 53$; HRP-polymer $\times 40$. (D) Loss of ATRX expression; HRP-polymer $\times 100$. (E) IDH1 polymerase chain reaction products (500 bp length) run on $2 \%$ agarose gel; ethidium bromide used as deoxyribonucleic acid (DNA) staining dye. (Bottom) Detection of DNA changes at R132 locus (R132H mutation, G > A) in two cases of IDH1R132H immunohistochemistry negative astrocytoma.

Recurrence of tumors occurred in $11(29.7 \%)$ cases at the mean \pm SD interval of $85 \pm 68.4$ days. In two of these patients, the tumors upgraded from grade II to grade III.

The IDH mutant and wild-type patients were compared for age, M:F ratio, location, and median survival by calculating $p$-value using chi-square test. The $p$-value was statistically significant $(p<0.001)$ for the age distribution showing difference in age at diagnosis between IDH mutant (32.5 years) and wild-type patients (38 years). There was no statistical significance for the gender distribution and location between the groups.
The patient survival period was calculated by KaplanMeier survival analysis. Median survival was 55.3 months and 22.2 months in IDH mutant and IDH wild-type cases, respectively. From the survival analysis, it was evident that patients with IDH mutations had better survival. Log-rank (Mantel-Cox) test was done for comparison of survival curves, which did not show any significance between these groups. This is shown in -Graphs $\mathbf{2}$ and $\mathbf{3}$.

The survival analysis showed that IDH wild-type grade III tumors had least median survival period with poor prognosis. 
SURVIVAL ANALYSIS

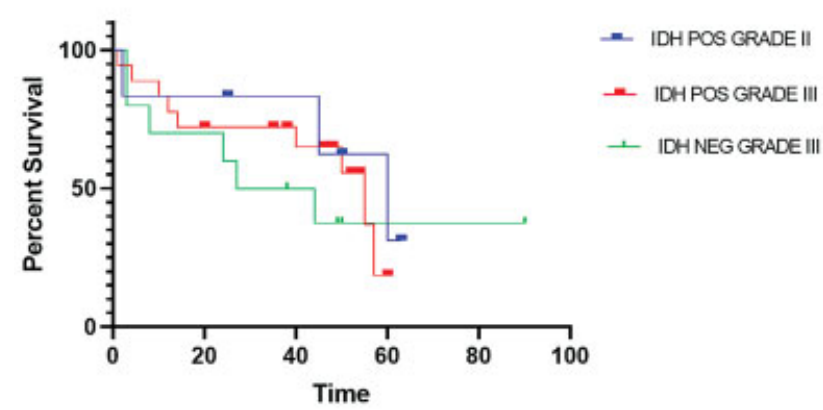

Graph 2 Kaplan-Meier survival analysis of IDH mutant grade II and III and IDH wild type grade III astrocytomas.

\section{Discussion}

DA is a common brain tumor mainly affecting young adults, and encompasses $\sim 11$ to $15 \%$ of all astrocytic brain tumors. In older adults over 40 , the age-adjusted annual incidence rate of astrocytic tumors is $6.8 / 100,000$ people. $6,11,12$ DA and AA are defined as tumors that lack the $1 p / 19 q$ codeletion and usually demonstrate loss of expression of ATRX and express mutant p53 protein.

IDH mutant and wild-type astrocytomas are clinically different tumors despite overlapping histological appearances. IDH mutations are the driving mutations in gliomagenesis, identified in 70 to $80 \%$ of DA. Majority of these mutations are IDH1 type, commonest being IDH1R132H. ${ }^{13}$ IHC for IDH1R132H is a surrogate for the mutation, and DNA sequencing is advocated only for the rare IDH1/2 mutations whenever diffuse gliomas are immunonegative for IDH1R132H. ${ }^{6}$

In this study, DA constituted $21.7 \%(51 / 234)$ of all the primary brain tumors in the study period. This is comparable to the study by Watanabe et $\mathrm{al}^{6}$ (29.5\%), however is lesser than that reported by Rajeswarie et $\mathrm{al}^{14}$ (47.5\%). The distribution of grades is similar to these two studies.

The mean age at presentation in our patients was $35.5 \pm 9.7$ years, which is comparable to that reported in other studies. ${ }^{11,12,14}$ IDH mutant gliomas have been found to have earliest age at diagnosis.

The most common presenting complaint was headache (70\%) followed by seizures (53\%), which is comparable to the study by Mu et al. ${ }^{11}$ Most common location in this study was frontal lobe (32\%) consistent with findings by Rajeswarie et $\mathrm{al}^{14}$ (41\% in grade II and $46.4 \%$ in grade III), Dong et al, ${ }^{15}$ and $\mathrm{Mu}$ et al. ${ }^{11}$

IHC with IDH1R132H was positive in $70.5 \%$ cases, of which $19.4 \%$ belonged to grade II and $80.5 \%$ belonged to grade III. Study by Mu et al ${ }^{11}$ observed IDH1R132H positivity in $77 \%$ of DA (19\% of grade II and $81 \%$ of grade III). Median age of IDH1R132H mutant astrocytoma was 32 years, which is comparable to other studies.

ATRX mutations have been reported either by sequencing or IHC in 45 to $67 \%$ of DA and 57 to $73 \%$ of AA. ${ }^{16-19}$ The mutations in ATRX are supposed to follow IDH mutations for astrocytic phenotype in a diffuse glioma. These are mutually

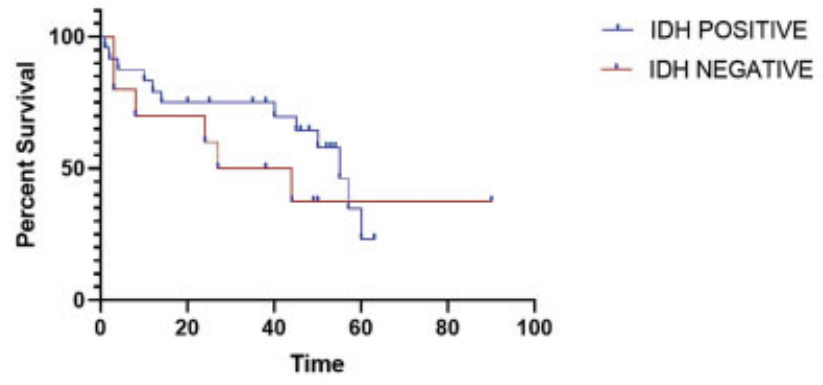

Graph 3 Kaplan-Meier survival analysis of IDH mutant and IDH wild type astrocytomas.

exclusive of $1 p 19 q$ codeletion. A surrogate IHC with loss of nuclear staining for ATRX in neoplastic cells, with retention in nonneoplastic cells serving as an internal positive control, is a reliable indicator of ATRX mutations. ${ }^{20}$ Inactivation of the adenosine triphosphate-dependent helicase ATRX has been linked to recombination-driven alternative telomere maintenance mechanisms and may provide glioma cells with unlimited proliferative capacity. ${ }^{21-23}$ In this study, $97.5 \%$ of IDH mutant astrocytoma showed loss of ATRX.

The WHO 2016 eliminated oligoastrocytoma as a distinct entity, since nearly all histologically defined oligoastrocytoma can be recategorized as oligodendroglioma or astrocytoma based on molecular features. ${ }^{24}$ We observed two cases of oligoastrocytoma that could be reliably classified as IDH mutant astrocytoma due to loss of ATRX and diffuse $p 53$ positivity. Another case of IDH mutant grade III astrocytoma with retained $A T R X$ did not show $1 p 19 q$ co deletion hence was classified as astrocytoma.

Tumorigenic Tp53 mutations have been reported to be present in $>50 \%$ of gliomas with astrocytic features, including 59 to $74 \%$ of DA and 53 to $65 \%$ of AA. ${ }^{4}$ In this study, diffuse and strong positivity for $p 53$ was observed in all cases of $I D H$ mutant astrocytoma. Grade II and III astrocytomas, which showed diffuse and strong positivity for $p 53$, constituted 72.8 and $91.6 \%$, respectively.

Pediatric DA is distinct clinically and molecularly from their adult counterparts. Clinical implications of BRAF alterations in these tumors and behavior of tumors with MYB/MYBL1/FGFR alterations are less clear. ${ }^{25}$ Although extremely common in adult diffuse glioma, IDH1/2 mutations are much less prevalent in pediatric gliomas. Previous reports suggested extremely low numbers of $I D H$ mutants in pediatric gliomas ranging from 0 to $17 \% .{ }^{25}$ In this study we noticed two pediatric patients with diffuse IDH mutant astrocytoma accounting for $2 / 40$ (5\%) of cases. These tumors are known to behave like adult IDH mutant astrocytoma. We did not identify any case of IDH wild type pediatric DA in our series.

Loss of the CDKN2A gene or p16 protein (the CDKN2A product) appears an ideal candidate for distinguishing the molecular phenotypes of WHO grade II and III IDH mutant gliomas. Studies showed that CDKN2A loss is associated with worse survival in astrocytoma. ${ }^{26}$ Available evidence from retrospective studies suggests that homozygous deletion of 
$C D K N 2 A / B$ is associated with shorter survival in patients with IDH mutant astrocytoma and that its presence corresponds to WHO grade IV clinical behavior. ${ }^{27,28}$ However, the molecular study was limited to identification of IDH mutation status, and other mutations that cause astrocytoma were not identified in this study.

Survival patterns for lower-grade astrocytoma remain poorly characterized. Several independent projects have demonstrated that histologic grading standards probably cannot distinguish prognoses for patients with $I D H$ mutant astrocytoma in the WHO grade II and III entities. ${ }^{29,30}$ On the contrary, some studies have concluded that traditional grading system is still able to stratify prognoses for these patients. ${ }^{31-33}$

In this study, all grade II and III DA patients with available follow-up received combined $54 \mathrm{~Gy}$ of IMRT and chemotherapy with temozolomide for a period ranging from 31 to 1,488 days and a mean of 374 days, while the study by Mu e tal ${ }^{11}$ observed that $48.6 \%$ received both chemotherapy and radiotherapy and $51.4 \%$ received only radiotherapy. Study by Dong et $\mathrm{al}^{15}$ observed $52.69 \%$ of grade II and $77.35 \%$ of grade III tumors receiving radiation.

The prognostic importance of IDH mutation is independent of other known prognostic factors, including age, grade, and MGMT methylation status. ${ }^{34}$ IDH mutations exhibit G-CIMP signature in glioma and the gliomagenesis has been attributed to the oncometabolite, 2-hydroxyglutarate (2-HG). This results in DNA and histone methylation. 2-HG is a part of DNA repair pathway and serves as an inhibitor of DNA repair enzymes as well as inhibits the homologous recombination DNA repair process. This helps in targeting the DNA repair enzymes by chemoradiotherapy giving a therapeutic benefit. IDH mutant tumors are also common in surgically amenable sites and gross total resection gives additional survival advantage. The second mutant protein, $A T R X$, is a chromatin-binding protein (SNF family) and the mutations result in telomere dysfunction. ATRX deficiency is associated with genomic instability that can induce p53dependent cell death. So p53 mutations in DA may enable tumor cell survival in the setting of ATRX loss. ${ }^{35}$

Studies showed that median survival of grade II and grade III astrocytomas is 7 to 10 years and 3.5 years, respectively. ${ }^{33}$ Pekmezci et al $^{32}$ observed median OS is better in patients with IDH mutant astrocytoma (9.3 years) than IDH wild-type astrocytoma (1.2 years). Another study identified that the median survival of $I D H$ wild-type gliomas was 1.7 years as against 6.3 years for $I D H$ mutant gliomas without $1 p 19 q$ codeletion. ${ }^{3}$ The similar was observed in our study with a median survival of 55.3 months (4.6 years) and 22.2 months (1.9 years) in IDH mutant and wild-type astrocytomas, respectively. However, this difference was not found to be statistically significant. This could perhaps be related to less number of IDH wild-type cases.

In a study by Shirahata et $a{ }^{33}$ it was identified that there was difference in survival of IDH mutant tumors. On followup, survival of $31.7 \%$ of IDH mutant glioblastomas and $42.6 \%$ of $I D H$ mutant AA was similar and this was associated with CDKN2A/B homozygous deletion. However, we did a similar comparison of median survival of $I D H$ mutant AA with $I D H$ mutant glioblastomas in the same period. We identified $61.7 \%$ of IDH mutant AA with median survival of 55.3 months and $7.3 \%$ of $I D H$ mutant glioblastomas with median survival of 12 to 15 months. However, we need to confirm these findings further by performing $C D K N 2 A / B$ deletion studies and expanding the sample size.

Limitations: The limitations of this study are retrospective study design. The follow-up details of all the patients were not available. Limited sample size of 51 patients is also a limitation for a complete statistical analysis.

Future Research Directions: There are advances in molecular genetics of diffuse gliomas including astrocytoma. The IDH mutant tumors are further classified based on $C D K N 2 A / B$ homozygous deletions. The tumors with these deletions are shown to have aggressive behavior even in $I D H$ mutant tumors. We plan to perform this deletion analysis and correlate with patient survival.

\section{Conclusion}

In conclusion, we observed that IHC with IDH1R132H, ATRX, and p53 is helpful in making an integrated diagnosis of astrocytic tumors as per the updated 2016 WHO classification of tumors. Other studies have also shown utility of IHC in subtyping the gliomas and our results are similar to these studies. Classification into definite molecular subgroups by sequencing to find out other variant IDH1 or IDH2 mutations is necessary according to WHO guidelines. IDH mutations correlate with survival and are good prognostic indicators in DA.

\section{Source of Funding}

Nil.

Conflict of Interest

Nil.

\section{References}

1 Louis DN, Perry A, Reifenberger G, et al. The 2016 World Health Organization Classification of Tumors of the Central Nervous System: a summary. Acta Neuropathol 2016;131(06):803-820

2 Brat DJ, Verhaak RG, Aldape KD, et al; Cancer Genome Atlas Research Network. Comprehensive, integrative genomic analysis of diffuse lower-grade gliomas. N Engl J Med 2015;372(26):2481-2498

3 Eckel-Passow JE, Lachance DH, Molinaro AM, et al. Glioma groups based on $1 \mathrm{p} / 19 \mathrm{q}$, IDH, and TERT promoter mutations in tumors. $\mathrm{N}$ Engl J Med 2015;372(26):2499-2508

4 Yan H, Parsons DW, Jin G, et al. IDH1 and IDH2 mutations in gliomas. N Engl J Med 2009;360(08):765-773

5 Hartmann C, Meyer J, Balss J, et al. Type and frequency of IDH1 and IDH2 mutations are related to astrocytic and oligodendroglial differentiation and age: a study of 1,010 diffuse gliomas. Acta Neuropathol 2009;118(04):469-474

6 Watanabe T, Nobusawa S, Kleihues P, Ohgaki H. IDH1 mutations are early events in the development of astrocytomas and oligodendrogliomas. Am J Pathol 2009;174(04):1149-1153

7 Hartmann C, Hentschel B, Wick W, et al. Patients with IDH1 wild type anaplastic astrocytomas exhibit worse prognosis than IDH1mutated glioblastomas, and IDH1 mutation status accounts for the unfavorable prognostic effect of higher age: implications for classification of gliomas. Acta Neuropathol 2010;120(06): 707-718 
8 Weller M, Weber RG, Willscher E, et al. Molecular classification of diffuse cerebral WHO grade II/III gliomas using genome- and transcriptome-wide profiling improves stratification of prognostically distinct patient groups. Acta Neuropathol 2015;129(05): 679-693

9 Brat DJ, Aldape K, Colman H, et al. cIMPACT-NOW update 3: recommended diagnostic criteria for "Diffuse astrocytic glioma, IDH-wildtype, with molecular features of glioblastoma, WHO grade IV". Acta Neuropathol 2018;136(05):805-810

10 Suri V, Jha P, Agarwal S, et al. Molecular profile of oligodendrogliomas in young patients. Neuro-oncol 2011;13(10):1099-1106

$11 \mathrm{Mu} \mathrm{L}, \mathrm{Xu}$ W, Li Q et al. IDH1 R132H mutation is accompanied with malignant progression of paired primary-recurrent astrocytic tumours. J Cancer 2017;8(14):2704-2712

12 Cohen AL, Holmen SL, Colman H. IDH1 and IDH2 mutations in gliomas. Curr Neurol Neurosci Rep 2013;13(05):345

13 Santosh V, Sravya P, Gupta T, et al. ISNO consensus guidelines for practical adaptation of the WHO 2016 classification of adult diffuse gliomas. Neurol India 2019;67(01):173-182

14 Rajeswarie RT, Rao S, Nandeesh BN, Yasha TC, Santosh V. A simple algorithmic approach using histology and immunohistochemistry for the current classification of adult diffuse glioma in a resource-limited set-up. J Clin Pathol 2018;71(04):323-329

15 Dong X, Noorbakhsh A, Hirshman BR, et al. Survival trends of grade I, II, and III astrocytoma patients and associated clinical practice patterns between 1999 and 2010: a SEER-based analysis. Neurooncol Pract 2016;3(01):29-38

16 Christians A, Adel-Horowski A, Banan R, et al. The prognostic role of IDH mutations in homogeneously treated patients with anaplastic astrocytomas and glioblastomas. Acta Neuropathol Commun 2019;7(01):156

17 Jiao Y, Killela PJ, Reitman ZJ, et al. Frequent ATRX, CIC, FUBP1 and IDH1 mutations refine the classification of malignant gliomas. Oncotarget 2012;3(07):709-722

18 Liu XY, Gerges N, Korshunov A, et al. Frequent ATRX mutations and loss of expression in adult diffuse astrocytic tumors carrying IDH1/IDH2 and TP53 mutations. Acta Neuropathol 2012;124(05): 615-625

19 Wiestler B, Capper D, Holland-Letz T, et al. ATRX loss refines the classification of anaplastic gliomas and identifies a subgroup of IDH mutant astrocytic tumors with better prognosis. Acta Neuropathol 2013;126(03):443-451

20 Ebrahimi A, Skardelly M, Bonzheim I, et al. ATRX immunostaining predicts IDH and H3F3A status in gliomas. Acta Neuropathol Commun 2016;4(01):60

21 Barthel FP, Wei W, Tang M, et al. Systematic analysis of telomere length and somatic alterations in 31 cancer types. Nat Genet 2017;49(03):349-357
22 Clynes D, Jelinska C, Xella B, et al. Suppression of the alternative lengthening of telomere pathway by the chromatin remodelling factor ATRX. Nat Commun 2015;6:7538

23 Heaphy CM, de Wilde RF, Jiao Y, et al. Altered telomeres in tumors with ATRX and DAXX mutations. Science 2011;333 (6041):425

24 Sahm F, Reuss D, Koelsche C, et al. Farewell to oligoastrocytoma: in situ molecular genetics favor classification as either oligodendroglioma or astrocytoma. Acta Neuropathol 2014;128(04): 551-559

25 Pollack IF, Hamilton RL, Sobol RW, et al; Children's Oncology Group. IDH1 mutations are common in malignant gliomas arising in adolescents: a report from the Children's Oncology Group. Childs Nerv Syst 2011;27(01):87-94

26 Reis GF, Pekmezci M, Hansen HM, et al. CDKN2A loss is associated with shortened overall survival in lower-grade (World Health Organization Grades II-III) astrocytomas. J Neuropathol Exp Neurol 2015;74(05):442-452

27 Brat DJ, Aldape K, Colman H, et al. cIMPACT-NOW update 5: recommended grading criteria and terminologies for IDH-mutant astrocytomas. Acta Neuropathol 2020;139(03):603-608

28 Aoki K, Nakamura H, Suzuki H, et al. Prognostic relevance of genetic alterations in diffuse lower-grade gliomas. Neuro-oncol 2018;20(01):66-77

29 Yoda RA, Marxen T, Longo L, et al. Mitotic Index Thresholds do not predict clinical outcome for IDH-mutant astrocytoma. J Neuropathol Exp Neurol 2019;78(11):1002-1010

30 Cimino PJ, Zager M, McFerrin L, et al. Multidimensional scaling of diffuse gliomas: application to the 2016 World Health Organization classification system with prognostically relevant molecular subtype discovery. Acta Neuropathol Commun 2017;5(01):39

31 Hasselblatt M, Jaber M, Reuss D, et al. Diffuse astrocytoma, IDHwildtype: a dissolving diagnosis. J Neuropathol Exp Neurol 2018; 77(06):422-425

32 Pekmezci M, Rice T, Molinaro AM, et al. Adult infiltrating gliomas with WHO 2016 integrated diagnosis: additional prognostic roles of ATRX and TERT. Acta Neuropathol 2017;133(06): 1001-1016

33 Shirahata M, Ono T, Stichel D, et al. Novel, improved grading system(s) for IDH-mutant astrocytic gliomas. Acta Neuropathol 2018;136(01):153-166

34 Sanson M, Marie Y, Paris S, et al. Isocitrate dehydrogenase 1 codon 132 mutation is an important prognostic biomarker in gliomas. J Clin Oncol 2009;27(25):4150-4154

35 Han S, Liu Y, Cai SJ, et al. IDH mutation in glioma: molecular mechanisms and potential therapeutic targets. $\mathrm{Br} J$ Cancer 2020; 122(11):1580-1589 